$7-2020$

\title{
Aggressive angiomyxoma in the ischiorectal fossa
}

Asra Wahid

Abdul Hakeem

Sadaf Khan

Follow this and additional works at: https://ecommons.aku.edu/pakistan_fhs_mc_mc

Part of the Life Sciences Commons, Oncology Commons, and the Surgery Commons 


\section{Aggressive angiomyxoma in the ischiorectal fossa}

Asra Wahid, ${ }^{1}$ Abdul Hakeem, ${ }^{2}$ Sadaf Khan ${ }^{3}$

\begin{abstract}
Aggressive Angiomyxoma (AA) is a soft tissue tumour that principally affects women of the reproductive age, primarily involving the pelvis and the perineum. Despite being slow growing, it is classified as aggressive due to its locally infiltrative nature and high recurrence. It is rare, often asymptomatic and is misdiagnosed. However, it has characteristic features on radiographic imaging. Complete surgical resection is the ideal approach to treat AA. We report a case of an aggressive angiomyxoma in a 17 year old female, located in the ischiorectal fossa and diagnosed incidentally on a magnetic resonance imaging (MRI). The lesion was excised without complication.
\end{abstract}

Keywords: Aggressive angiomyxoma, Mesenchymal neoplasm, Perineal tumour.

\section{DOI: https://doi.org/10.5455/JPMA.30242}

\section{Introduction}

Aggressive angiomyxoma is a slow growing mesenchymal neoplasm. ${ }^{1}$ It arises primarily in the deep soft tissues of the pelvis, perineum of the reproductive age women and in the inguinoscrotal region in the men. The tumour has a locally infiltrative nature. It has a high rate of local recurrence (70\%) but lacks metastatic potential.2,3 Clinically, patients are often asymptomatic and may be misdiagnosed. The tumour is usually large $(>10 \mathrm{~cm})$ and is discovered during pelvic examination or on a radiographic imaging. $1,4,5$ It has a lobulated appearance with a soft rubbery consistency and may infiltrate into surrounding tissues. ${ }^{3}$ On magnetic resonance imaging $(\mathrm{MRI})$, it has a characteristic internal low intensity swirled appearance on both $\mathrm{T} 1$ and $\mathrm{T} 2$ weighted images. ${ }^{4}$ Histologic examination reveals a sparsely cellular tumour consisting of spindle and stellateshaped cells in a myxoid background studded with blood vessels. ${ }^{2}$ Treatment modality includes a wide local surgical excision with a long term follow-up. ${ }^{3,6}$ We report a case of a 17-year-old female being evaluated for a year

1Fourth Year Medical Student, 2,3Department of Surgery, Aga Khan University Hospital, Karachi, Pakistan.

Correspondence: Sadaf Khan. Email: Sadaf.khan@aku.edu long history of lower back pain who was incidentally found to have a mass in the ischiorectal fossa on a MRI. It is unique, because it is a rare and slowly progressing disease with vague symptoms, difficult diagnosis and no definite treatment guidelines. In our case, a successful complete surgical resection with clear margins was performed which is generally the preferred treatment. This could add to the literature of successful cases and can potentially help define treatment in the future. Informed consent was taken from the patient for writing the report.

\section{Case Presentation}

A 17-year-old female with an unremarkable medical and surgical history presented to the clinic with lower back pain. It was associated with a burning sensation localized to the lower back along with numbness radiating to the legs. Clinical examination was unremarkable. She was managed conservatively with analgesics and muscle relaxants but had persistent symptoms, she presented first time at Aga Khan University Hospital, Karachi on 8th August 2017.

A pelvic MRI was obtained, which did not identify any spinal pathology. However, it showed a $1.9 \times 2.1 \mathrm{~cm}$ welldefined rounded lesion in the right ischiorectal fossa closely abutting right obturator internus with an intact fat planes laterally and anteromedially, extending to the levatorani muscle. The lesion was hypointense on T1 and

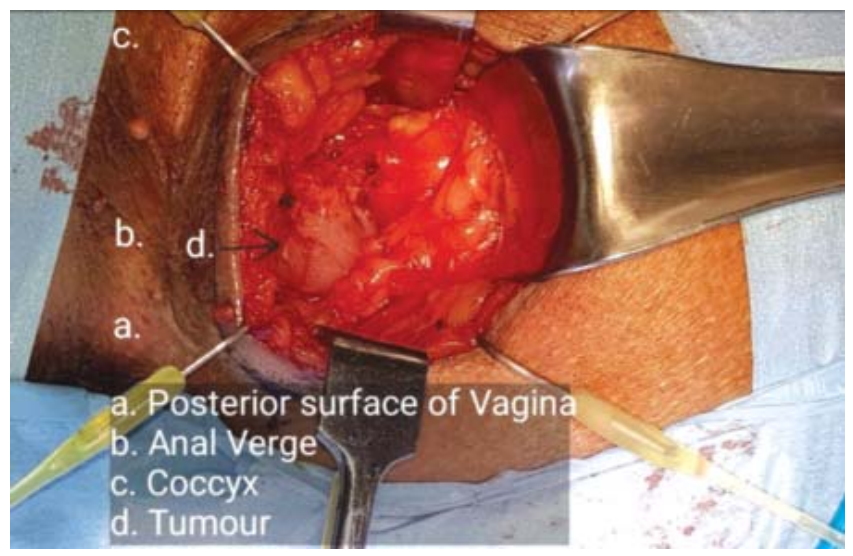

Figure-1: View of the structures within the perianal region before excision of the tumour. 


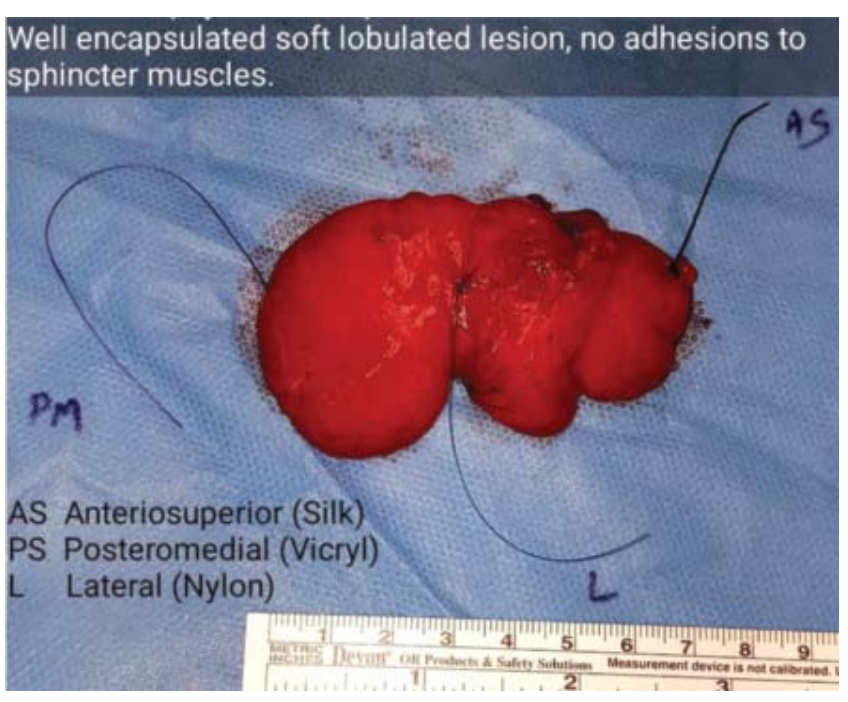

Figure-2: Gross examination of the excised tumour.

hyperintense on T2-weighted images, with subtle postcontrast enhancement. She was referred for surgical evaluation 5 months after her initial MRI and was advised a repeat $M R I$. It revealed an increase in the size $(4 \times 3 \mathrm{~cm})$ of the lesion. Differential diagnosis after a repeat MRI included a lipoma and a liposarcoma. A computed tomography (CT) scan guided core biopsy was suggestive of an aggressive angiomyxoma. A decision for surgical excision was made.

Bowel was prepared with rectal enemas. After the administration of general anaesthesia, patient was placed in a prone-jackknife position. A $6 \mathrm{~cm}$ crescent incision was placed over the right ischiorectal fossa. Dissection was continued proximally into the space using electrocautery as shown in Figure-1. With help of a manual palpation, the lesion was identified and sharply dissected and care was taken not to breach the capsule. The lesion was well circumscribed without any signs of surrounding infiltration or extension into the rectum medially or in the levatorani superiorly. On excision, the lesion was noted to be soft with a smooth surface, measuring $7 \times 4.5 \times 2 \mathrm{~cm}$ (Figure-2).

On histopathology, the cut surface was grey-white. Microscopic examination revealed a neoplastic lesion comprising of haphazard arrangement of spindle shaped cells in a myxoid background. Individual cells had elongated vesicular nuclei, inconspicuous nucleoli, scant eosinophilic cytoplasm and interspersed thin strands of collagen. Vessels showed adventitial thickening with perivascular lymphocytic cuffing with presence of smooth muscle cells. Occasional mitotic figures were seen but necrosis was absent. The tumour's histological features were consistent with an aggressive angiomyxoma. Immunohistochemistry showed positive desmin, CD 34 and ER signals while S-100 and an alpha smooth muscle actin ( $\alpha$ - SMA) were negative.

No post-surgery complications were seen; wound site was managed with dressing and stitches were removed within 2 weeks. Patient complained of constipation postoperatively for 2 weeks which responded to medications and dietary manipulation. Follow up scan after 6 and 12 months revealed no recurrence of the disease.

\section{Discussion}

Aggressive Angiomyxoma (AA), a slow growing and benign mesenchymal tumour occurs in women in their 40s and 50s.2,4 It usually occurs in the female pelvis and the perineum especially, the vulva. We the report a case of a 17-year-old female in the ischiorectal fossa. In men, AA is rarely reported, usually involving the inguinoscrotal region. Male to female ratio is $1: 6.6 .3$ In a study reported from Pakistan, it was seen to comprise of $10 \%$ of the benign tumours of the vulva. ${ }^{7}$

AA usually causes displacement of adjacent organs typically without invasion. However, due to its locally infiltrative and aggressive nature it can cause invasion during longstanding growth. ${ }^{6}$ These tumours have a high rate of local recurrence varying from $36-70 \%$, usually within the first two years. However, it is treated by reexcision with a $1 \mathrm{~cm}$ margin. 1,6

Patients are often asymptomatic or present with a painless mass. However, our patient had a year long history of nonspecific back pain which had resolved by the time she came for the surgery. Due to their location, AAs are usually large and measure more than $10 \mathrm{~cm}$ at the time of the diagnosis. Frequently the large, deep seated pelvic component of the aggressive angiomyxomas in relation to the pelvic diaphragm is not appreciated on clinical examination and can often be missed. 8

Clinically, differential diagnosis of AA includes myxoma, myxoid liposarcoma, pelvic fibromatosis, levator hernia, bartholin cyst, labial cyst, gartner duct cyst or lipoma. ${ }^{1,5}$ The main histologic differential consideration would be an angiomyofibroblastoma.

Imaging modalities that assist with diagnosis include CT scan, MRI and pelvic digital subtraction angiography (DSA). ${ }^{9}$ Sonographic imaging shows a hypoechoic or a cystic mass. ${ }^{4}$ Angiography typically shows a hyper vascular mass. ${ }^{9}$ On CT scan, the tumour has a distinct border with minimal hypodensity compared to muscle. On MRI, both T1-weighted and T2-weighted images, mass 
has areas of "swirled" linear low-intensity signal suggesting a fibrovascular stroma. On T1-weighted images, the tumour is isointense compared to a muscle. T2-weighted images show a hyperintense tumour due to high water content and loose myxoid matrix. In our patient, we report similar findings on a MRI. ${ }^{4}$

On gross assessment, tumours are usually not encapsulated and have sharp margination in some areas while blending gradually with surrounding soft tissue in others. Areas of congested blood vessels, haemorrhage or fibrosis may be present. ${ }^{9}$ Our patient's tumour had a similar appearance (Figure-2), on inspection it was a pink/gray mass with a rubbery texture and a gelatinous glistening cut surface. Microscopic findings in our patient are consistent with those reported in the literature.

Resection margins are usually positive due to the tumour's tendency to infiltrate local tissues. Almost all AA on immunohistochemistry show positive vimentin and desmin signals with a negative S-100 signals. The positive rates of CD34, smooth muscle actin (SMA), a-SMA, oestrogen receptor (ER) or progesterone receptor (PR) have been reported many times in the literature 4 . We report positive desmin, CD 34, ER signals along with a negative S-100 and a-SMA signals. Recent molecular assessments indicate that certain genetic alterations appear to have a role in the pathogenesis of this tumour and may be useful markers for microscopic residual disease.

Complete surgical resection of the lesion must be considered, as done in our case. Partial resection is considered when a high surgical morbidity or a mutilating surgery is anticipated. This can be bolstered with adjunct therapies, including arterial embolization, hormonal treatment or radiotherapy. ${ }^{10}$

There are no definite guidelines on the postoperative management of AA. Since the recurrence rate is as high as $72 \%$ and undiagnosed occurrences are associated with morbidity, various authors have recommend periodic assessment comprising of physical examination and a MRI after the surgical resection. Follow-up for up to 15 years has been described. ${ }^{10}$ At 1 year our patient showed no evidence of recurrent disease. A definite prognosis of this would require a long term follow up, which is a limitation in this report.

\section{Conclusion}

Angiomyxoma is a rare tumour that is often missed due to an unremarkable clinical examination. It is common in women of mid-30s, however, it can also present in teenagers, as in our case. No definite guidelines exist regarding its management but complete surgical resection is the key treatment. However, regular long follow-ups are needed to assess the recurrence.

Disclaimer: None to declare.

Conflict of Interest: None to declare.

Sources of Funding: None to declare.

\section{References}

1. Kura MM, Jindal SR, Khemani UN. Aggressive angiomyxoma of the vulva: An uncommon entity. Indian Dermatol Online J 2012; 3:128-30. doi: 10.4103/2229-5178.96712.

2. Amezcua CA, Begley SJ, Mata N, Felix JC, Ballard CA. Aggressive angiomyxoma of the female genital tract: a clinicopathologic and immunohistochemical study of 12 cases. Int J Gynecol Cancer 2005; 15:140-5. DOI: 10.1111/j.1048-891x.2005.15015.x

3. Hastak MS, Raghuvanshi SR, Sahu S, Vyankatesh A, Ramraje SN, Ranjan A. Aggressive angiomyxoma in men. J Assoc Physicians India 2008; 56:373-5.

4. Benson JC, Gilles S, Sanghvi T, Boyum J, Niendorf E. Aggressive angiomyxoma: case report and review of the literature. Radiol Case Rep 2016; 11:e332-5. DOI: 10.1016/j.radcr.2016.10.006

5. Surabhi VR, Garg N, Frumovitz M, Bhosale P, Prasad SR, Meis JM. Aggressive angiomyxomas: a comprehensive imaging review with clinical and histopathologic correlation. AJR Am J Roentgenol 2014; 202:1171-8. doi: 10.2214/AJR.13.11668.

6. Geng J, Cao B, Wang L. Aggressive angiomyxoma: an unusual presentation. Korean J Radiol 2012; 13:90-3. doi: 10.3348/kjr.2012.13.1.90.

7. Perveen S. Benign tumours of the vulva. J Coll Physicians Surg Pak 2004;14:537-9. DOI: 05.2005/JCPSP.537539

8. Fetsch JF, Laskin WB, Lefkowitz M, Kindblom LG, Meis-Kindblom JM. Aggressive angiomyxoma: a clinicopathologic study of 29 female patients. Cancer 1996; 78:79-90. DOI: 10.1002/(SICI)10970142(19960701)78:1<79::AID-CNCR13>3.0.CO;2-4

9. Kumar S, Agrawal N, Khanna R, Khanna A. Aggressive angiomyxoma presenting with huge abdominal lump: A case report. Cases J 2008; 1:131. doi: 10.1186/1757-1626-1-131.

10. Han-Geurts IJ, van Geel AN, van Doorn L, M den Bakker, Eggermont AM, Verhoef C. Aggressive angiomyxoma: multimodality treatments can avoid mutilating surgery. Eur J Surg Oncol 2006; 32:1217-21. DOI: 10.1016/j.ejso.2006.06.008. 